\title{
Beetle luciferases with naturally red- and blue-shifted emission
}

\author{
César Carrasco-López ${ }^{1}$ (D), Juliana C Ferreira ${ }^{1}$, Nathan M Lui ${ }^{1}$ (D), Stefan Schramm¹, Romain Berraud-Pache², \\ Isabelle Navizet ${ }^{2}$ (D), Santosh Panjikar ${ }^{3,4}$ (D), Panče Naumov ${ }^{1}$, Wael M Rabeh ${ }^{1}$ (])
}

\begin{abstract}
The different colors of light emitted by bioluminescent beetles that use an identical substrate and chemiexcitation reaction sequence to generate light remain a challenging and controversial mechanistic conundrum. The crystal structures of two beetle luciferases with red-and blue-shifted light relative to the green yellow light of the common firefly species provide direct insight into the molecular origin of the bioluminescence color. The structure of a blue-shifted green-emitting luciferase from the firefly Amydetes vivianii is monomeric with a structural fold similar to the previously reported firefly luciferases. The only known naturally red-emitting luciferase from the glow-worm Phrixothrix hirtus exists as tetramers and octamers. Structural and computational analyses reveal varying aperture between the two domains enclosing the active site. Mutagenesis analysis identified two conserved loops that contribute to the color of the emitted light. These results are expected to advance comparative computational studies into the conformational landscape of the luciferase reaction sequence.
\end{abstract}

DOI 10.26508/Isa.201800072 | Received 23 April 2018 | Revised 5 August 2018 | Accepted 6 August 2018 | Published online 16 August 2018

\section{Introduction}

The dazzling flashes of bioluminescent light communicated by fireflies have inspired scientists, writers, artists, and laymen for centuries. Beyond its visual appeal, bioluminescence is an irreplaceable bioanalytical tool for in vivo imaging, monitoring of cell proliferation, studies into protein folding and secretion, environmental research, and food quality control. At the core of this natural phenomenon lies a fundamental process of energy transduction by luciferase enzymes that convert the chemical energy stored within the ground-state substrate (luciferin) to an excited, emissive state of the product (oxyluciferin) by a spin-forbidden process (Johnson \& Shimomura, 1972; DeLuca, 1976). Although beetle luciferase systems share identical substrates and chemical reaction sequence, they emit a range of different colors from yellow green $\left(\lambda_{\max } \approx 560 \mathrm{~nm}\right)$, which is typical for common firefly species, such as the North American firefly Photinus pyralis $\left(G_{p p}\right)$ and the Japanese firefly Luciola cruciata $\left(G_{L c}\right)$, to orange and even red $\left(\lambda_{\max }=\right.$ 590-623 nm) from certain click beetles and railroad worms (Viviani et al, 1999, 2011; Ugarova \& Brovko, 2002). The molecular origin of the different colors of light emitted by different luciferases remains the most elusive mechanistic aspect of this photochemistry and has been continuously debated since the 1970s. In the absence of structural information on WT luciferases that emit light other than green, several mechanisms have been advanced and subsequently refuted. Current mechanistic models that are based on experimental and computational analysis on available green-emitting luciferase structures remain inconclusive (Hosseinkhani, 2011).

Here, we describe the first crystal structures of two rare WT luciferases from Brazilian beetles that emit light with exceptional colors; a luciferase from the head lanterns of the glow-worm P. hirtus (Coleoptera: Phengodidae), the only known luciferase that naturally emits red light $\left(\lambda_{\max }=623 \mathrm{~nm}\right.$; $\left.R E_{\mathrm{ph}}\right)$, and a green-emitting luciferase from the firefly $A$. vivianii (Coleoptera: Lampyridae) that displays a blue-shifted emission relative to common firefly luciferases $\left(\lambda_{\max }=\right.$ $538 \mathrm{~nm}$ at pH 8; $\mathrm{GB}_{\mathrm{Av}}$ ) (Viviani et al, 1999, 2008, 2011). Biochemical and structural analyses of the two luciferases, combined with computational modeling, provide the best insight yet into the relationship between the structure and color of light emitted by beetle luciferases.

\section{Results}

Structural determination of the red-emitting luciferase from P. hirtus

The crystal structure of WT RE $E_{p h}$ was determined at low resolution by molecular replacement from two different crystal forms in the space groups $P 1$ and $P 3_{1} 21$ at resolution of $3.05 \AA$ and $3.60 \AA$, respectively (Table S1). Both crystal forms presented good-quality electron density maps, which were improved by the non-crystallographic symmetry of both unit cells (Fig S1A and B). Unlike previously reported luciferases that are exclusively monomeric (Conti et al, 1996; Franks et al, 1998;

${ }^{1}$ New York University Abu Dhabi, Abu Dhabi, United Arab Emirates ${ }^{2}$ Laboratoire Modélisation et Simulation Multi Echelle, MSME UMR 8208 CNRS, Université Paris-Est, Marne-la-Vallée, France ${ }^{3}$ Australian Synchrotron, Clayton, Australia ${ }^{4}$ Department of Biochemistry and Molecular Biology, Monash University, Melbourne, Australia 
Nakatsu et al, 2006; Auld et al, 2010; Cruz et al, 2011; Sundlov et al, 2012; Kheirabadi et al, 2013; Branchini et al, 2017), in the P3,21 crystal form, $\mathrm{RE}_{\mathrm{Ph}}$ exists as tetramer, although in the $P 1$ crystal form it is an octamer in the asymmetric unit (Figs $1 \mathrm{~A}$ and $\mathrm{B}, \mathrm{S} 2 \mathrm{~A}$, and $\mathrm{B}$ ). The $\mathrm{N}$-terminal domains in the octamer core structure are assembled as a tetramer of dimers and packed over dimer and tetramer interfaces, and the C-terminal domains point outward. This assembly accounts for the structural flexibility and increased thermal motion of the C-terminal domains which is apparent from the residual electron density. The inability to model the C-terminal domain of firefly luciferases in certain crystal conditions, as result of its high flexibility, has been previously shown (Auld et al, 2010; Thorne et al, 2012; Kheirabadi et al, 2013). Only one out of the four C-terminal domains was observed in the density maps of the $P 3_{1} 21$ crystal form and none of the eight C-terminal domains of the $R E_{P h}$ octamer could be resolved in the $P 1$ crystal form (Supplementary Note 1). The RE $E_{p h}$ structure is consistent with the $\alpha / \beta$-fold of other beetle luciferases and the substrate-binding pocket in each of the monomers is located between the larger ( $\mathrm{N}$-terminal) and smaller (C-terminal) domains. A displacement of the C-terminal domain can open and close the active site in a conformational rearrangement that is purportedly triggered by binding of the substrates (Nakatsu et al, 2006).

In the structure of $\mathrm{RE}_{\mathrm{Ph}}$, each dimer within the octamer is stabilized by multiple hydrogen bonds between amino acid residue R11 from one of the monomers and $\mathrm{Y} 26, \mathrm{Y} 30$, and N179 from the other monomers, which extend over a $C_{2}$ axis across the dimer interface (Fig 1C). The interactions across the dimer interface are strong electrostatic interactions with two contact points contributed by R11 from each monomer (Fig 1C). With the low resolution of the $\mathrm{RE}_{\mathrm{Ph}}$ structure, site-directed mutagenesis clearly confirms these interface interactions, where the single mutation (R11A) was sufficient to disrupt the octamer of $\mathrm{RE}_{\mathrm{Ph}}$ and resulted exclusively monomers in solution (Fig S2C and D). On the other hand, the hydrophobic interactions between M152, Y153, and F162 from two dimers contribute to the weak overall interactions between the dimers over the tetramer interface (Fig 1D). As confirmed by mutagenesis and sizeexclusion chromatography, the interactions across the tetramer interface are weaker relative to the interaction of the individual monomers over the dimer interface. The WT RE $E_{\mathrm{Ph}}$ exists as a tetramer in solution (Supplementary Note 2). Consequently, mutations on the tetramer interface, which include single (F162A) and double (Y153A and F162A) mutants, produced only dimers in solution (Fig S2C). Overall, the emission of WT RE $E_{p h}$ remains unaffected by interface mutations that result in fragmentation of the RE $E_{P h}$ octamer into dimers and monomers; we therefore conclude that the red emission of $\mathrm{RE}_{\mathrm{ph}}$ is not a result of its quaternary structure. Instead, the red light is intrinsic to the structural fold of the $R E_{\mathrm{Ph}}$ monomer and originates from the specific packing and microenvironment of its active site.

\section{Structural determination of the blue-shifted green emission luciferase from $A$. vivianii}

To identify structural features that are important in the color tuning mechanism, the crystal structure of $\mathrm{GB}_{\mathrm{Av}}$ luciferase with a blue-shifted

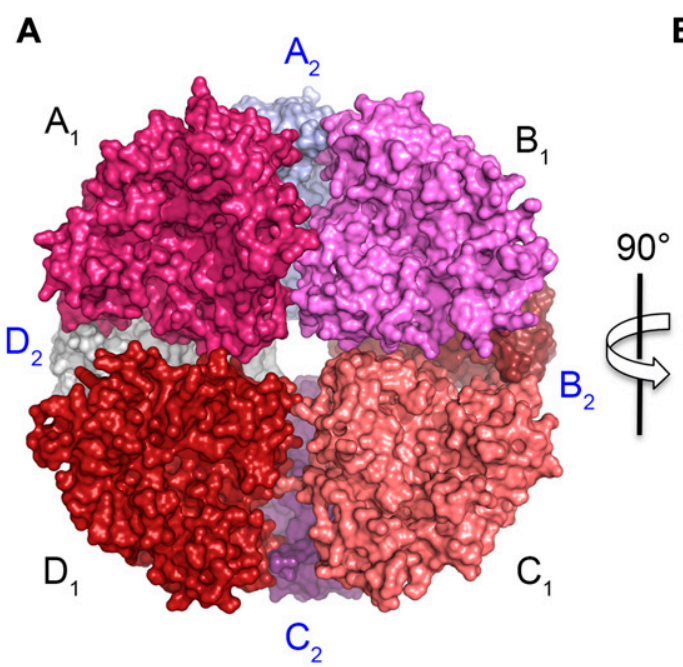

B

C Dimer Interface

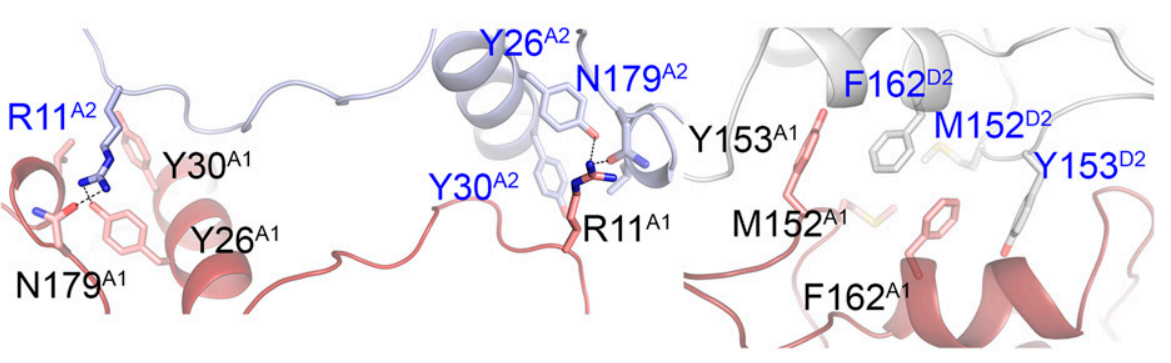

Figure 1. Crystal structure of the naturally redemitting luciferase from $P$. hirtus $\left(R_{P h}\right)$.

(A) Front view of the partial octameric conformation found in the P1 crystal form (only the N-terminal domains were observed in the electron density maps). (B) Lateral view of the octamer that highlights the surface interactions between the monomers. (C) Closeup view and interactions across the dimer interface between monomers A1 (red) and A2 (blue). The interacting residues are shown as stick models with matching colors as monomers $A 1$ and $A 2$ in panels $(A, B)$. The two surfaces are related to each other by a $C_{2}$ axis. The broken lines show the interactions between residues R11, N179, and Y26. Mutation R11A disrupted the octamer to give monomers in solution. (D) Close-up view of the tetramer interface across the dimers, which are assembled as an octamer (monomer A1 is dark red and monomer D2 is white). The interactions between the two surfaces are predominantly hydrophobic interactions between Y153, M152, and F162 from both dimers. Similar to the dimer interface, the surfaces at the tetramer interface are related by a $C_{2}$ axis. Single and double mutations at residues Y153 and F162 generated exclusively dimers in solution. 
green emission of $l_{\max }=538 \mathrm{~nm}$ at $\mathrm{pH} 8$ was determined for comparison with the RE $E_{P h}$ (Fig 2B and C). The emission of $G_{A v}$ is at higher energy relative to the well-studied luciferases $G_{L c}$ (Nakatsu et al, 2006) and $G_{P p}$ (Conti et al, 1996) that emit green-yellow light. The $\mathrm{GB}_{\mathrm{Av}}$ crystals diffract to a resolution of $1.9 \AA$ when free of substrate and crystallize in a space group $P 2_{1} 2_{1} 2_{1}$ with two independent $\mathrm{GB}_{\mathrm{Av}}$ monomers in the asymmetric unit (Fig S1E and F and Table S1). Although the N-terminal domains of the two $\mathrm{GB}_{\mathrm{Av}}$ molecules are very similar (root-mean-square deviation [RMSD] value of $0.1 \AA$ calculated on all backbone atoms), their C-terminal domains have different conformations with an RMSD value of $2.1 \AA$ (Fig S3A and B). Thus, although the luciferase in both molecules can be considered as being in its "open" conformation, the luciferase with the smaller aperture is maybe an intermediate between the "open" and "closed" conformations (Figs 2B, S3A, and B), the latter being attributed to the structure of $G_{L c}$ in complex with the reaction products (Nakatsu et $\mathrm{al}$, 2006). Indeed, a superposition of the $\mathrm{N}$-terminal domains of the "open" conformations of $\mathrm{GB}_{\mathrm{Av}}$ and $\mathrm{RE}_{\mathrm{Ph}}$ with the green-emitting luciferase $G_{L c}$ in complex with oxyluciferin and AMP (Protein Data Bank
[PDB] code: 2D1R), which is in its "closed" state (Nakatsu et al, 2006), indicates a movement of the C-terminal domain of about $10 \AA$ to $30 \AA$ between the two states to open/close the active site (Fig S4). The pronounced flexibility of the C-terminal domain of $\mathrm{GB}_{\mathrm{Av}}$ was further examined by classical molecular dynamics simulations in which the C-terminal domain required 5 to 15 ns to shuffle between the two conformations. Together with the structural data, these results confirm the pronounced mobility of the C-terminal domain of beetle luciferases, which is capable of reversible opening and closing of the active site through two catalytic conformations during the bioluminescence reaction. The two catalytic conformations are stimulated by rotation on the C-terminal domain of firefly luciferases (Sundlov et al, 2012). Notably, the structures of both $G_{A v}$ and $R E_{P h}$ have wider openings than $G_{p p}$ luciferase devoid of substrates or products (PDB codes: $1 \mathrm{LCl}$ and 5DV9; Figs $2 \mathrm{~A}$ and $\mathrm{B}$, and S4A) (Conti et al, 1996; Wu et al, 2017). The aperture of the active site with an angle of $\sim 125^{\circ}$ between axes crossing the center mass of each domain ( $P 3,21$ crystal form) shows that $\mathrm{RE}_{\mathrm{Ph}}$ is the most open conformation
A

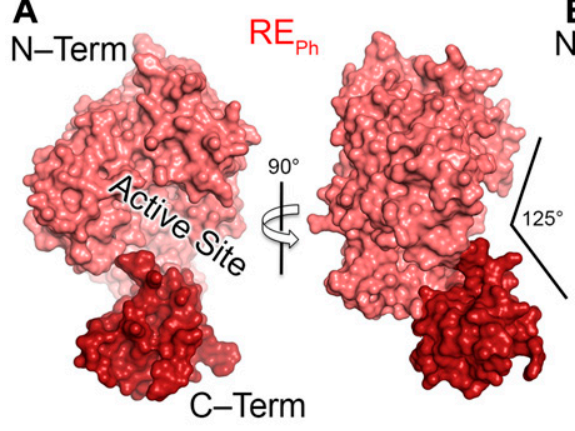

C

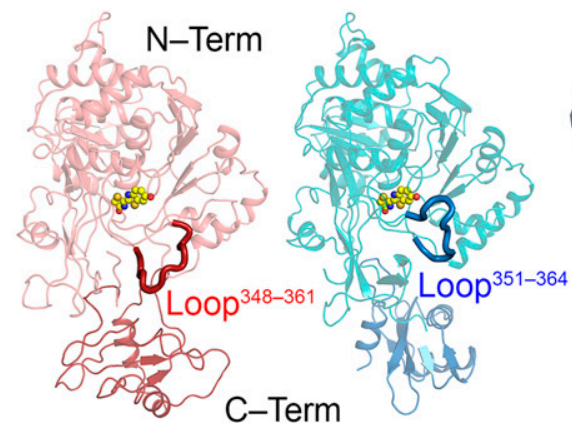

.
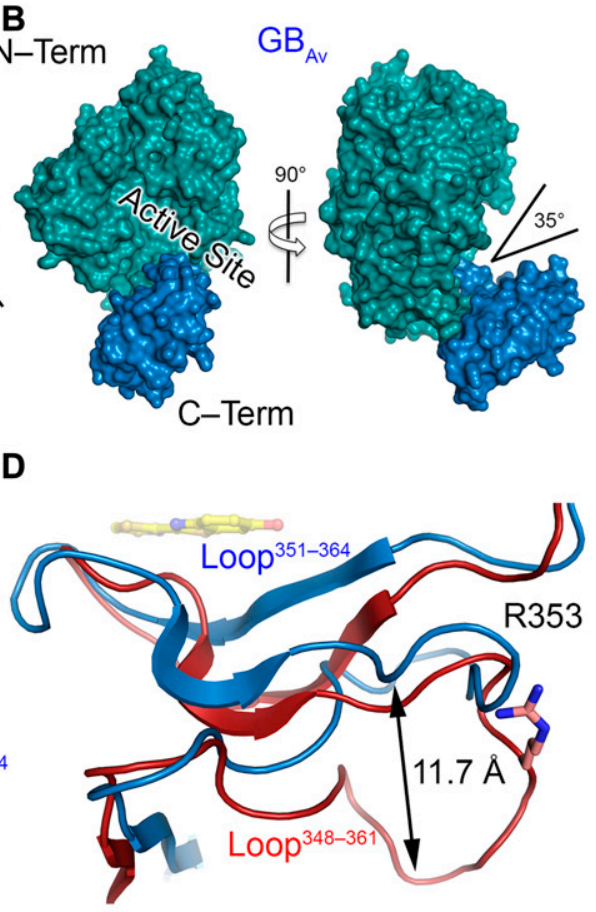

E

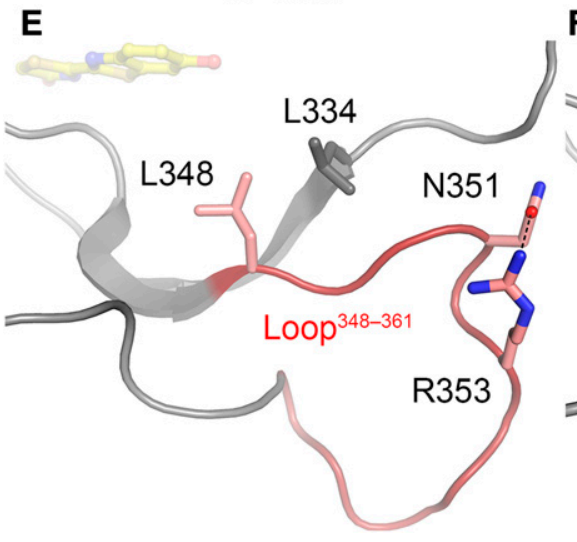

$\mathbf{F}$

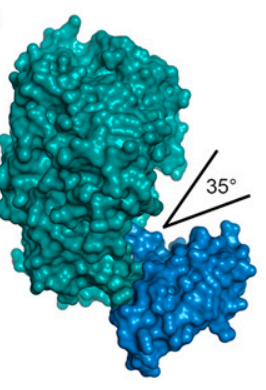

(a)

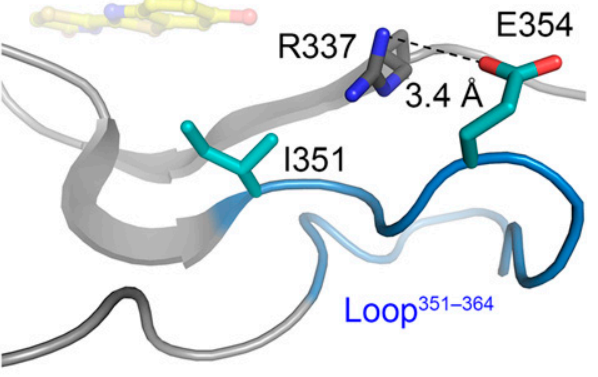

Figure 2. Analysis of the structures of red-emitting $\mathrm{RE}_{\mathrm{Ph}}$ and blue-shifted green-emitting $\mathrm{GB}_{\mathrm{Av}}$ luciferases. (A) Full-length structure of molecule $B$, the only monomer with a complete $\mathrm{C}$-terminal domain of $\mathrm{RE}_{\mathrm{Ph}}$ in the $P 3_{1} 21$ crystal form (the structures of the other three monomers in this crystal lack the C-terminal domain, which could not be observed in the difference electron density maps). The conformation of $\mathrm{RE}_{\mathrm{Ph}}$ has the largest aperture between the $\mathrm{N}$-terminal ("N-Term") and

C-terminal ("C-Term") domains among the luciferases with known crystal structures. (B) Structure of one of the two monomers in the asymmetric unit of $\mathrm{GB}_{\mathrm{AV}}$ (the monomer of $\mathrm{GB}_{\mathrm{AV}}$ with a larger aperture is shown here; the angle of the aperture of the other monomer is $30^{\circ}$ ). The structural packing of both $\mathrm{GB}_{\mathrm{Av}}$ monomers is less open relative to $R E_{\mathrm{ph}}$. The RMSD value of the superimposed monomers is $0.22 \mathrm{~A}$ based on all $\alpha$-carbons in the structure (the deviations were prominent in the C-terminal domain, with RMSD of 2.1 A). Identical conformations were found for the N-terminal domain, with RMSD based on the $\alpha$-carbons of $0.08 \AA$. (C) The loose packing of loop ${ }^{348-361}$ (red) in the $\mathrm{N}$-terminal domain of $\mathrm{RE}_{\mathrm{ph}}$ relative to the tight packing of loop ${ }^{351-364}$ (blue) in the N-terminal domain of $\mathrm{GB}_{\mathrm{Av}}$ (see Fig S6). (D) The shift of loop p $^{348-361}$ (red) in $\mathrm{RE}_{\mathrm{Ph}}$ relative to loop ${ }^{351-364}$ (blue) in $\mathrm{GB}_{\mathrm{Av}}$ by superposition of the two monomers based on the $\alpha$-carbons of the whole structures (Fig S6A). R353 (pink sticks) is the only known insertion in the REph sequence. (E) Natural substituted residues found in loop (48-361 $^{34}$ (pink) of $\mathrm{RE}_{\mathrm{ph}}$ close to the active sites are shown in gray and pink sticks. (F) The relatively conserved residue counterparts in loop ${ }^{351-364}$ (blue) of $\mathrm{GB}_{\mathrm{Av}}$ are shown in gray and blue sticks. In panels $\mathrm{D}, \mathrm{E}$, and $\mathrm{F}$, the reaction product (oxyluciferin, shown with yellow sticks) is shown by superimposing the structures reported here with the structure of $\mathrm{G}_{\mathrm{LC}}$ in complex with oxyluciferin (PDB code: 2D1R). 
among the known luciferase structures, which corresponds to the high mobility of its C-terminal domain. This feature indicates that the flexibility of the C-terminal domain may play a role in tuning the color of light emitted.

\section{Loop ${ }^{351-364}$ is important for the green and red emission of $\mathrm{GB}_{\mathrm{Av}}$ and $\mathrm{RE}_{\mathrm{Ph}}$}

Detailed analyses of the structures of $\mathrm{RE}_{\mathrm{Ph}}, \mathrm{GB}_{\mathrm{Av}}$, and $\mathrm{G}_{\mathrm{Lc}}$ revealed multiple amino-acid residues that may be directly involved in determining the color of emitted light (Supplementary Note 3). The most remarkable structural feature is the conserved loop $351-364$ in $\mathrm{GB}_{\mathrm{Av}}$ (which corresponds to loop ${ }^{348-361}$ in $\mathrm{RE}_{\mathrm{ph}}$ ) that is located in the $\mathrm{N}$-terminal domain at the edge of the active site (Figs $2 \mathrm{C}$ and $\mathrm{D}$, and $\mathrm{S} 5$ ). In addition, the presence of the only known insertion in beetle luciferases (R353 in RE $\mathrm{ph}_{\text {; }}$ Fig S5), the proximity of loop ${ }^{351-364}$ to the active site and several key substitutions around the benzothiazole-binding region, which were previously found to have an impact in the color tuning (Viviani et al, 2007), drove us to investigate the relevance of loop ${ }^{351-364}$ in the color-tuning mechanism. The loop ${ }^{348-361}$ in $\mathrm{RE}_{\mathrm{Ph}}$, albeit at low resolution, was modeled in the electron-density map (Fig S1C). In $\mathrm{GB}_{\mathrm{Av}}$, loop ${ }^{351-364}$ is tightly held by strong ionic interactions between E354 on the loop and R337 on the N-terminal domain (Figs 2F, S6, and S7). Residue K358 on the opposite side of the loop also interacts with D427 and D429 from the terminal section of the N-terminal domain (for figure clarity, these interactions are not shown in Fig 2F). The ionic interactions on both sides of loop $\mathrm{p}^{351-364}$ fix its position relative to the enzyme backbone. This stability is reflected in the low RMSD value for the loop backbone atoms of only $0.3 \AA$ between $G_{A v}$ and $G_{L c}$. These interactions are absent in $\mathrm{RE}_{\mathrm{Ph}}$ owing to the replacement of R337 and E354 in $\mathrm{GB}_{\mathrm{Av}}$ with L334 and N351 in REph, respectively (Figs 2E, S5, and $\mathrm{S} 6 \mathrm{~A})$. The absence of these strong interactions with the enzyme core (represented by R337) increases the mobility of analogous loop ${ }^{348-361}$ in $\mathrm{RE}_{\mathrm{Ph}}$, as reflected in the higher RMSD value of $2.3 \AA$ for the respective atoms between the $\mathrm{RE}_{\mathrm{ph}}$ monomers. Notably, $\mathrm{RE}_{\mathrm{ph}}$ is also the only beetle luciferase with an additional residue, R353, in loop ${ }^{348-361}$ (Figs 2E and S5). Although insertion of R353 in green-emitting luciferases including, $\mathrm{GB}_{\mathrm{Av}}$ (Fig 3D and Table S2), red-shifts the color that is emitted (Tafreshi et al, 2007; Alipour et al, 2009), its deletion from the redemitting $\mathrm{RE}_{\mathrm{ph}}$ as demonstrated here and previously does not affect the red emission (Viviani et al, 2007).

Additional biochemical and computational analyses were performed to assess the effects of R337 on the emission color because our and others' (Viviani et al, 2007; Viviani et al, 2016) mutation experiments indicated that it has an important role in determining the emitted color (Fig 3 C and D and Supplementary Notes 3 and 4 and Tables S3, S4, and S5). Mutation of R337L in green-emitting GB $B_{A v}$ red-shifted its emission by $42 \mathrm{~nm}$, from 538 to $580 \mathrm{~nm}$ (Fig 3B). The theoretical calculations reproduced the observed trend and estimated a red shift of $\approx 60 \mathrm{~nm}$, from 535 to $577 \mathrm{~nm}$ (Table S4, snapshot 4). A second mutation, I351L, did not affect the emission energy of R337L, which shows the dominating effect of R337 (Table S3). These results confirm that the interactions of $\mathrm{R} 337$ with loop $\mathrm{p}^{351-364}$ in $\mathrm{GB}_{\mathrm{Av}}$ are critical for the emission of green light. We anticipate that this effect is more general for green-emitting luciferases and an analogous mutation that disrupts these interactions would shift their green emission $\left(\lambda_{\max } \approx 560 \mathrm{~nm}\right)$ and thereby decrease the emitted energy $\approx 40-60 \mathrm{~nm}$ to generate emission of red light ( $\approx 600-620 \mathrm{~nm})$. Thus, it appears that the absence of strong interactions of L334 in red-emitting luciferase RE $\mathrm{E}_{\mathrm{Ph}}$ (analogous to R337 in $\mathrm{GB}_{\mathrm{Av}}$ ) is critically important for its red emission. In support of this hypothesis, replacement of L334 (mutation L334R) in red-emitting $\mathrm{RE}_{\mathrm{Ph}}$ blue-shifted the light of this luciferase by $18 \mathrm{~nm}$, from 623 to $605 \mathrm{~nm}$ without a significant change in light intensity (Fig 3A). Similarly, the double mutant L334R/L348I of RE emissions from 623 to $600 \mathrm{~nm}$, although the single mutation L348I did not affect the emission maximum, which confirms that L334 in $\mathrm{RE}_{\mathrm{Ph}}$ is the key residue that determines the low energy of its emission. Nevertheless, these mutants still emit red light. We hypothesize that synergistic effects that involve residues outside the active site could stabilize the closed state and shift the emission further to the green.

\section{Loop ${ }^{523-530}$ only contributes to the green emission of $\mathrm{GB}_{\mathrm{Av}}$}

A second highly conserved loop in beetle luciferases at the end of the C-terminal domain, loop ${ }^{523-530}$ in $\mathrm{GB}_{\mathrm{Av}}\left({ }\right.$ loop ${ }^{521-528}$ in $\mathrm{RE}_{\mathrm{Ph}}$ ), is also relevant to the aperture of the enzyme (Figs S4 and S5). Despite the low resolution of the $\mathrm{RE}_{\mathrm{ph}}$ structure, the high-quality electron density map allowed to model this loop region in the $P 33_{1} 21$ crystal form (Fig S1D), where rotation and displacement of loop $p^{523-530}$ by $\sim 10 \AA$ closes the active site and facilitates interactions of key residues with the substrates. In the closed state of $G_{L C}, K 526$ interacts with T292 and D468 of the $\mathrm{N}$ - and C-terminal domains, respectively (Fig S4E). Upon closure of the active site, T527 and K529 of $\mathrm{GB}_{\mathrm{Av}}$ are shifted to interact with the substrate in accordance with their catalytic roles in the bioluminescence reaction (Branchini et al, 2000, 2004, 2005). To assess the role of the conformation of the c-terminal domain on the emission from the excited state of the product, a complementary umbrella classical molecular dynamics simulation was performed on $\mathrm{GB}_{\mathrm{Av}}$ in which $\mathrm{K} 524$ of the $\mathrm{C}$-terminal domain was constrained to interact with the backbone of $\mathrm{G} 311$ of the N-terminal domain to close the active site in $\mathrm{GB}_{\mathrm{Av}}$ (Figs S5, S8, and S9 and Supplementary Note 4). The results revealed that rotation of the C-terminal domain between the open and closed states is not related to energetic barrier. Moreover, the bioluminescence emission calculated for the closed state $(530 \pm 10 \mathrm{~nm})$ is in strong agreement with the experimental value of $538 \mathrm{~nm}$ (Table S6). Thus, the presence of loop ${ }^{523-530}$ inside the active site of the luciferase is required for the natural bioluminescence color of $\mathrm{WT} \mathrm{GB}_{\mathrm{Av}}$.

We were able to discern with sufficient accuracy the structure of loop ${ }^{523-530}$ in both independent molecules in the crystal of $\mathrm{GB}_{\mathrm{Av}}$, in which the open conformation is stabilized by interactions between T527 and K529 of loop ${ }^{523-530}$, and D422 and D436 of the N-terminal domain (Fig S4C). The structural integrity of loop $p^{523-530}$ is maintained by interaction between $\mathrm{K} 524$ and the backbone of G528. The mutant K524A in $100 p^{523-530}$ of $\mathrm{GB}_{\mathrm{Av}}$ exhibited a red shift of $12 \mathrm{~nm}$ with minimum decrease in its thermodynamic stability (Figs $3 \mathrm{C}$ and D, S10B, E, F, and G-I, and Supplementary Note 3). The emission of the same mutant (K522A) of $R E_{P h}$ remained unaltered, even though its thermodynamic stability decreased (Figs 3C, S10A, C, D, and G-I). With the exception of T525A in $\mathrm{RE}_{\mathrm{Ph}}$, which retained $\approx 40 \%$ of its emission intensity, other mutants in loop ${ }^{523-530}$, which include T527A and $\mathrm{K} 529 \mathrm{~A}$ of $\mathrm{GB}_{\mathrm{Av}}$, and T525A and K527A of RE $E_{\mathrm{Ph}}$, suppressed the WT enzyme emission completely. 
A

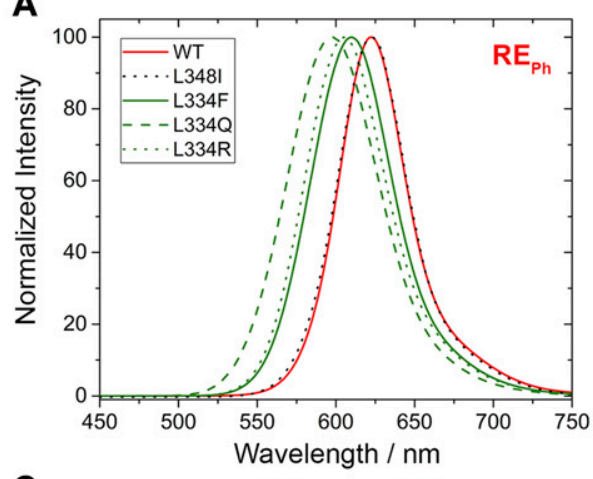

C

$$
\text { C }
$$

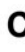

B

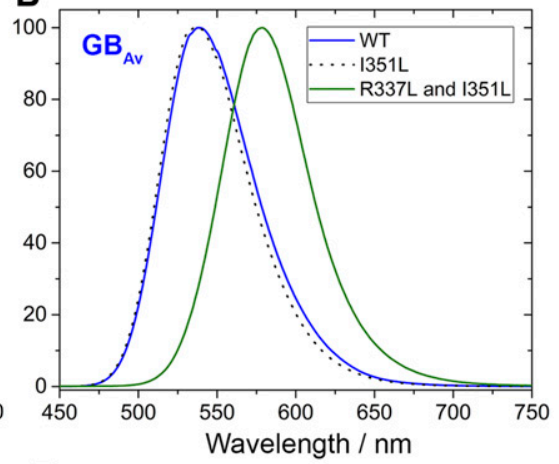

E

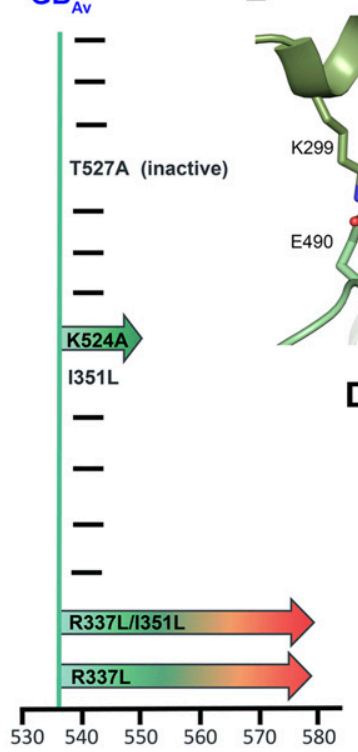

$\mathrm{GB}_{\mathrm{Av}}$

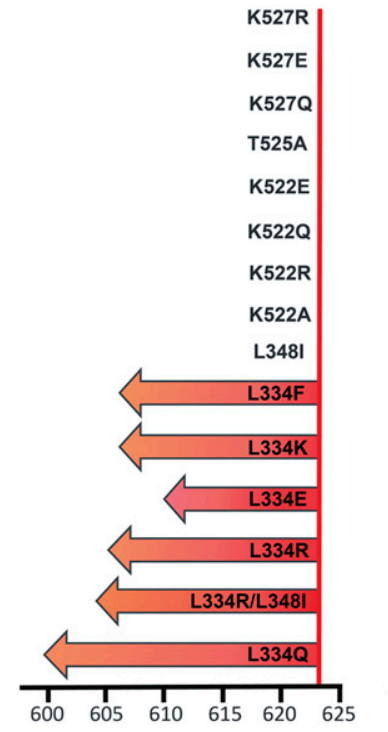

Wavelength / $\mathrm{nm}$

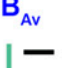

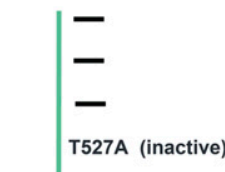

T527A (inactive) $\mathrm{nm}$

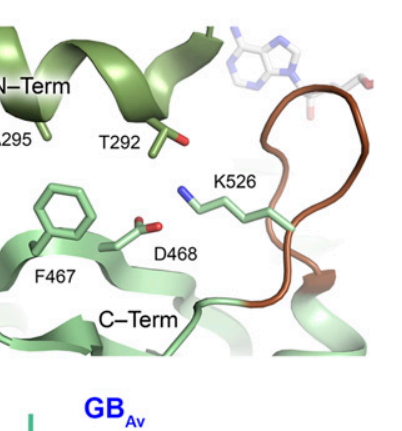

Figure 3. Normalized bioluminescence emission spectra and kinetics of WT and mutants of luciferases $\mathrm{RE}_{\mathrm{Ph}}$ and $\mathrm{GB}_{\mathrm{Av}}$.

(A) At pH 8.0, the emission from WT RE $E_{\mathrm{Ph}}$ with $\lambda_{\max }=623$ $\mathrm{nm}$ was blue-shifted between 600 and $610 \mathrm{~nm}$ in mutants of L334. (B) At pH 8.0, GB $B_{A v}$ emission at $\lambda_{\max }=$ $538 \mathrm{~nm}$ for the WT enzyme was red-shifted to $580 \mathrm{~nm}$ in double mutant R337L/I351L. Single mutant I351L did not alter the emission of $\mathrm{GB}_{\mathrm{Av}}$ ( (C, D) Schematic of the experimental (C) and calculated (D) data for mutationinduced shifts of emissions of $\mathrm{GB}_{\mathrm{Av}}$ and $\mathrm{RE}_{\mathrm{Ph}}$ (Tables S2, S3, S4, and S5). The vertical $y$-axis is set at the WT emission of $\mathrm{GB}_{\mathrm{Av}}$ or $\mathrm{RE}_{\mathrm{Ph}}$. Each arrow represents a mutant (labeled inside the arrow) that shifts the color from the WT emission and points in the direction of change of the emitted color. The tip of the arrow is a qualitative representation of the color shift and the maximum emission wavelength. The mutations that did not affect the color emitted by the WT luciferase are labeled immediately next to the $y$-axis. (E) Residues at the interface (green sticks) between the $\mathrm{N}$ - and C-terminal domains in the closed conformation of $\mathrm{G}_{\mathrm{LC}}$. Previous mutations on residues at this interface (E490 and $\mathrm{F} 467$ ) and mutant $\mathrm{K} 524 \mathrm{~A}$ in $\mathrm{GB}_{\mathrm{AV}}\left(\mathrm{K} 526\right.$ in $\mathrm{G}_{\mathrm{LC}}$ ) reported here red-shifted the color between 10 and $15 \mathrm{~nm}$.
The relevance of the $\mathrm{C}$-terminal domain in the color-tuning mechanism was further explored by introducing mutation K524A, which is distant from the active site in both the open and closed conformations (Fig 3E). The slightly decreased thermodynamic stability of this mutant indicates minor destabilization of the interactions of $\mathrm{K} 524$ with T290 and D466 (as seen in the structure of $\mathrm{GB}_{\mathrm{Av}}$ ), which are at the interface between the $\mathrm{N}$ - and C-terminal domains in the closed state (Fig S4E and Supplementary Note 3). This result is similar to the red shift in emission of other mutations that have been introduced previously in the C-terminal domain and are located on the same interface (Fig 3). This interface between the $\mathrm{N}$ - and C-terminal domains includes polar and hydrophobic interactions that, when disrupted, are known to shift the color to red by $\approx 10-15 \mathrm{~nm}$ without affecting the general fold of the enzyme (Modestova \& Ugarova, 2016).

\section{Discussion}

The structural determination of the only naturally red-emitting $R E_{\mathrm{Ph}}$ has, for the first time, revealed an oligomeric structure for a beetle luciferase, whereas others are all exclusively monomeric. Although oligomerization is possible in solution, it is not critical for the emission of red light; instead, the low emission energy is inherent to the structure of the monomer and the microenvironment of the active site. Although co-crystals with either substrate could not be achieved, the structures of $R E_{P h}$ with red emission and $G_{A v}$ with blue-shifted green emission provide insight into the effects that conformational changes and active site microenvironment have on the color of the light emitted by luciferases. Furthermore, two conserved segments, loop ${ }^{351-364}$ in $\mathrm{GB}_{\mathrm{Av}}$ and loop ${ }^{348-361}$ in $\mathrm{RE}_{\mathrm{Ph}}$, were identified by mutagenesis to have a profound effect on the emission of both enzymes.

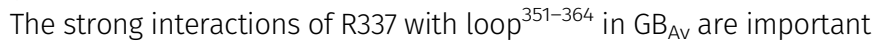
for its green emission. Similarly, mutations that altered the interactions with loop $^{348-361}$ blue-shift the emission of $\mathrm{RE}_{\mathrm{Ph}}$ from $623 \mathrm{~nm}$, for the WT, to $605 \mathrm{~nm}$ in the L334R mutant. The second conserved segment, loop ${ }^{523-530}$ in $\mathrm{GB}_{\mathrm{Av}}$ or loop ${ }^{521-528}$ in $\mathrm{RE}_{\mathrm{Ph}}$, was found to be important for the emission of $\mathrm{GB}_{\mathrm{Av}}$ only, and substitutions in loop ${ }^{521-528}$ did not alter the red emission of $\mathrm{RE}_{\mathrm{Ph}}$. These results provide direct insight into the molecular origin of the diverse colors emitted by different beetle luciferases and are the key to solving one of the most difficult conundrums in bioluminescence research. 


\section{Materials and Methods}

\section{Materials}

Unless mentioned otherwise, the chemicals were from SigmaAldrich. The synthesis of full-length genes and oligonucleotide DNA primers, DNA sequencing, and, in few cases, site-directed mutagenesis were carried out by GenScript USA Inc. Restriction enzymes were purchased from New England Biolabs Inc., and KOD DNA polymerase and dNTP mix were from EMD Millipore.

\section{Cloning, expression, and purification}

The genes of the natural red-emitting luciferase from $P$. hirtus ( $R E_{p h}$; railroad worm) and the green-emitting luciferase with blue-shifted emission $\left(G_{A v}\right)$ from A. vivianii were synthesized by Genscript (GenScript USA Inc.). The DNA sequences, based on the previously reported proteins sequences (Viviani et al, 1999, 2011), were optimized for Escherichia coli expression and designed to be subcloned into a pET26b-derived bacterial expression vector that contains an $\mathrm{N}$-terminal domain SUMO fusion protein tag (Champion pET SUMO system, Thermo Fisher Scientific) by using Nhel and Xhol as restriction sites. Further subcloning was performed into selected pET28-derived systems by using Nhel-HindIII restriction sites ( $\mathrm{RE}_{\mathrm{Ph}}$ ) and BamHI-HindIII $\left(G_{A v}\right)$. The best overexpression results were obtained for $\mathrm{GB}_{\mathrm{Av}}$ cloned into the pET28a+ system (Novagen), whereas the RE $E_{P h}$ expression was improved by using the $\mathrm{pET} 28 \mathrm{MHL}$ system (provided by the Structural Genomics Consortium). Most of the point mutations were introduced by site-directed mutagenesis (Edelheit et al, 2009), whereas others were ordered from Genscript (all point mutations used to destabilize the octamer).

The luciferase constructs were introduced by transformation into E. coli BL21-CodonPlus-RIL (Stratagene). A single colony was used to inoculate Luria broth that contains kanamycin $(100 \mathrm{mg} / \mathrm{l}$ ) and chloramphenicol (50 mg/l) and grown overnight at $37^{\circ} \mathrm{C}$ with vigorous shaking (preinocules). The inoculated cultures (usually 4-6 liters for each construct) were grown at $37^{\circ} \mathrm{C}$ until the $A_{600}$ reached 0.2 . At this point, the temperature was lowered to $28^{\circ} \mathrm{C}$ until an $A_{600}$ of 0.3 was achieved, and the luciferases expression was then induced overnight $\left(\right.$ at $\left.15^{\circ} \mathrm{C}\right)$ by adding IPTG $(0.2 \mathrm{mM})$. The cells were harvested by centrifugation at 12,000 $\mathrm{g}$ for $10 \mathrm{~min}$ in an Avanti J26XPI centrifuge (Beckman Coulter Inc.), then resuspended in lysis buffer (Tris [100 mM], pH 7.8, NaCl [500 mM], glycerol [10\%], imidazole [5 mM], $\beta M E$ [3 $\mathrm{mM}$ ], and protease inhibitor cocktail from Sigma-Aldrich: P8849), lysed by sonication on ice, and centrifuged again at $40,000 \mathrm{~g} \mathrm{rpm}$ for $30 \mathrm{~min}$ at $4^{\circ} \mathrm{C}$. The supernatants were loaded at a rate of $1 \mathrm{ml} / \mathrm{min}$ onto a ProBond Nickel-Chelating Resin (Life Technologies) previously equilibrated with binding buffer (Tris [100 mM], pH 7.8, NaCl [500 mM], glycerol [10\%], imidazole [5 mM], and $\beta M E[3 \mathrm{mM}])$ at $4^{\circ} \mathrm{C}$. The columns were washed with 10 column volumes (cv) of binding buffer, followed by $15 \mathrm{cvs}$ of washing buffer (Tris [100 mM], pH 7.8, NaCl [250 mM], glycerol [10\%], $\beta M E$ [3 mM], and imidazole [50 mM]). Recombinant luciferases were eluted by using elution buffer (Tris [100 mM], pH 7.8, NaCl [500 mM], glycerol [10\%], $\beta M E$ [3 mM], and imidazole [300 mM]). Finally, the proteins were loaded onto a HiLoad Superdex S200 size-exclusion filtration column (GE Healthcare), attached to an AKT $\Delta$ purifier core system (GE Healthcare), and pre-equilibrated with filtration buffer (Hepes [20 mM], pH 7.8, NaCl [500 mM], glycerol [10\%], and TCEP [1 mM]). The final protein peaks were collected and concentrated to $\approx 5-10 \mathrm{mg} / \mathrm{ml}$ (measured by absorbance) and their purity was analyzed by using SDS-PAGE.

\section{Crystallization}

A high-throughput approach was used to explore initial crystallization conditions for $\mathrm{RE}_{\mathrm{Ph}}(7.2 \mathrm{mg} / \mathrm{ml})$ and $\mathrm{GB}_{\mathrm{Av}}(5.6 \mathrm{mg} / \mathrm{ml}) \mathrm{lu}$ ciferases that were previously purified. Commercially available conditions from screens PACT suite, JCSG (Qiagen), INDEX, and Crystal Screen 1 and 2 (Hampton Research), were tested by using a vapor diffusion method on sitting drops. A total of $75 \mu \mathrm{l}$ of each condition was dispensed into a 96-well SD2 Molecular Dimensions (MD2) plate by a Bravo robot (Agilent Technologies). A volume of $250 \mathrm{nl}$ of protein was mixed (1:1) with each condition by using a Honeybee $X 8$ robot (Isogen Life Science). The plates were sealed, incubated at $18^{\circ} \mathrm{C}$, and checked regularly under the microscope. Along with the free enzymes, several complexes that include the enzyme and ATP (in presence of $\mathrm{MgCl}_{2}$ ), enzyme and luciferin, and enzyme and ATP and luciferin (supplemented with $\mathrm{MgCl}_{2}$ ) were tested at different ligand concentrations (0.1-5.0 mM).

The hits obtained for both enzymes were systematically explored to improve the initial crystals by making $2 \mathrm{D}$ variations of the $\mathrm{pH}$ and all the components (precipitant and additives) in 96-well plates, and manually in MRC Maxi 48-Well Crystallization Plate (Swissci). The improved conditions were scaled to $1.5 \mu \mathrm{l}$ drops (protein/ precipitant $=2: 1$ ) and bigger single crystals were obtained for both $\mathrm{GB}_{\mathrm{Av}}$ and $\mathrm{RE}_{\mathrm{Ph}}$ (free enzymes). The final crystallization conditions were sodium citrate (1.6 M), pH 6.5, supplemented with $\mathrm{MgCl}_{2}$ (200 mM) for $\mathrm{GB}_{\mathrm{Av}}$, and polyethylene glycol 3350 (19-21\%), bis-tris propane $(100 \mathrm{mM}), \mathrm{pH} 6.0$, supplemented with ammonium sulfate (200 mM) for $R E_{p h}$.

\section{X-ray data collection and structure determination}

The crystals of both proteins were fast-frozen in liquid nitrogen. Only the crystals of $\mathrm{RE}_{\mathrm{Ph}}$ were soaked into a cryoprotectant solution (crystallization solution supplemented with 15-20\% glycerol) as the final crystallization condition for $\mathrm{GB}_{\mathrm{Av}}$ crystals (sodium citrate [1.6 m], pH 6.5) worked as a cryoprotectant solution. The diffraction data were collected by using synchrotron radiation at the Swiss Light Source (beamline X06DA) with a Pilatus 2M detector (Dectris) and at the MX2 beamline from the Australian Synchrotron with a Quantum $315 r$ Detector (ADSC). Routinely, $360^{\circ}$ were collected for each crystal with an oscillation range between 0.1 and $0.5^{\circ}$ and exposure time of 0.1-1 s per image, depending on the quality of crystals. Several datasets were collected for $\mathrm{GB}_{\mathrm{Av}}$ (free enzyme) up to $1.9 \AA$ (Fig 1B), others for RE $E_{p h}$ (free enzyme) up to $3.1 \AA$ at X06DA and up to $3.6 \AA$ at MX2 beamline (Australian Synchrotron). Other datasets were collected for crystals of $\mathrm{GB}_{\mathrm{Av}}$ soaked with ATP (up to $2.2 \AA$ ). The crystals of REph are in the triclinic space group $P 1$ (parameters: $a=105.70 \AA$; $\left.b=121.17 \AA ; c=129.44 \AA ; \alpha=61.86^{\circ} ; \beta=68.35^{\circ} ; \gamma=74.17^{\circ}\right)$ and trigonal P3,21 (parameters: $a=b=119.100 \AA ; c=351.402 \AA ; \alpha=\beta=90.00^{\circ}$; $y=120.00^{\circ}$ ), whereas crystals of $\mathrm{GB}_{\mathrm{Av}}$ are in the orthorhombic space 
group $P 22_{1} 2_{2} 2_{1}$ (parameters: $a=94.28 \AA ; b=110.53 \AA ; c=122.07 \AA$; $\alpha=\beta=\gamma=90^{\circ}$ ).

For the $P 1$ crystal form of $\mathrm{RE}_{\mathrm{Ph}}$, two isomorphous datasets were merged to obtain a good dataset at $3.05 \AA$ (Table S1), which was used to solve the structure. The datasets were indexed and integrated by using XDS (Kabsch, 2010) and iMosflm (Battye et al, 2011) and scaled with Scala (Evans, 2006) from the CCP4 program suite (Winn et al, 2011). The protein structures were phased by molecular replacement using the program Molrep (Vagin \& Teplyakov, 1997) within the molecular replacement protocol of Auto-Rickshaw (Panjikar et al, 2005, 2009). The $\mathrm{N}$-terminal domain of the Japanese firefly structure (PDB code: 2D1R) was used as a template. Refinement of the structures was carried out with the PHENIX program (Afonine et al, 2012). As a result of differences in the conformation of the C-terminal domains, this part of the structure was manually built in both molecules of $\mathrm{GB}_{\mathrm{AV}}$ found in the asymmetric unit, and later in the only complete monomer of $\mathrm{RE}_{\mathrm{Ph}}$ using the program Coot (Emsley et al, 2010). An iterative process of manual building and refinement with the PHENIX program (Afonine et al, 2012) was applied to both structures. Data processing and refinement statistics are summarized in Table S1. The final models presented good stereochemistry parameters and $R$ and $R_{\text {free }}$ values. Also, both $R E_{p h}$ crystal forms showed good-quality electron density maps for their low resolution (Fig S1A and B). The figures of the structures were generated with PyMol (DeLano, 2002). Structure factors and atomic coordinates are deposited in the Protein Data Bank (PDB accession codes: 6AAA, 6ABH, and 6AC3).

\section{Differential scanning calorimetry (DSC)}

Calorimetric analyses for the WT proteins and mutants were performed by using a Nano-DSC (TA Instruments). The samples and buffers were degassed under vacuum for 15 min with stirring at $10^{\circ} \mathrm{C}$. The concentrations of all the proteins were adjusted to $0.5 \mathrm{mg} / \mathrm{ml}$. A total volume of $300 \mu \mathrm{l}$ of each degassed sample in Hepes (20 mM; pH 7.8) and $\mathrm{MgCl}_{2}(10 \mathrm{mM}$ ) was loaded into the sample cell. For measurements with ligands, ATP and firefly luciferin (Gold Biotechnology) were added to both, the sample and reference cell in the same concentration $(5 \mu \mathrm{M})$. The samples were heated at a scan rate of $1^{\circ} \mathrm{C} / \mathrm{min}$ from 10 to $80^{\circ} \mathrm{C}$ at $3 \mathrm{~atm}$. Before loading the samples, background scans were obtained by loading degassed buffer (with or without substrates) in both the reference and samples cells and heated at the same rate. The enthalpies of the transitions $\left(\Delta H_{\text {cal }}\right)$ were estimated by calculating the area under the thermal transition after subtracting the blank and fitting a baseline by using Nano Analyzer software provided by the manufacturer.

\section{Computational analysis}

The closed state of $\mathrm{GB}_{\mathrm{Av}}$ was modeled with classical MD and quantum mechanics (QM)/ MM calculations. To integrate the oxyluciferin in its keto form and protonated adenosine 5'-monophosphate nucleotide $(\mathrm{AMPH})$ molecules in the active site of $\mathrm{GB}_{\mathrm{Av}}$, manual docking was performed based on the position of these two molecules in the structures of the North American firefly, P. pyralis (PDB code: 4G37), and the Japanese firefly, L. cruciata (PDB code: 2D1R). All calculations were performed on one of the molecules (chain $B$ ) in the $\mathrm{GB}_{\mathrm{Av}}$ structure.

The residues were protonated by using Leap from Amber14 suite of the program (Case et al, 2017). The contentious cases, especially for histidines, were resolved by computing their $\mathrm{p} \mathrm{K}_{\mathrm{a}}$ with the $\mathrm{H++}$ program (DeLano, 2002) to have a neutral charge for the system. Depending on the model considered, we selected to doubleprotonate (i.e., one hydrogen on each nitrogen of the side chain, which resulted in a positively charged residue) the following histidines for luciferases in complex with oxyluciferin and AMPH:

(1) $\mathrm{GB}_{\mathrm{Av}}$-open with, $\mathrm{GB}_{\mathrm{Av}}$-closed, and $\mathrm{GB}_{\mathrm{Av}}$-closed-1347L: the doubly protonated histidines are 5, 23, 42, 72, 305, 404, and 426.

(2) $\mathrm{GB}_{\mathrm{Av}}$-closed-R337L: the doubly protonated histidines are 5, 23, 42, 72, 305, 404, 426, and 456.

(3) $\mathrm{GB}_{\mathrm{Av}}$-closed-insert-R356: the doubly protonated histidines are $5,23,42,72,405$, and 427.

AMPH was included with a single negative charge, and oxyluciferin was modeled in its phenolate-keto form, with a single negative charge.

Classical dynamics simulations were performed with Amber14 to obtain several snapshots for further QM/MM optimization. The model was solvated with TIP3P water molecules within a cubic box by ensuring a solvent shell of at least $15 \AA$ around the solute. The resulting system contained $\approx 28,000$ water molecules and 90,000 atoms in total. The AMBER99ff was used to model the residues of the protein. The AMPH and the emitter (oxyluciferin) were described by using parameters developed by the Navizet group (Navizet et al, 2010, 2011; Chen et al, 2011; Anandakrishnan et al, 2012). The parameters were not fully optimized for the excited state, so the oxyluciferin structure was first obtained by QM/MM optimization of the first singlet excited state and frozen in its excited state conformation during the whole dynamics simulation. The system was heated from 100 to $300 \mathrm{~K}$ in $20 \mathrm{ps}$. Then, under isothermal-isobaric ensemble (NPT) conditions with $T=300 \mathrm{~K}$ and $P=1 \mathrm{~atm}$, a 10-ns dynamic with periodic boundary conditions was realized with a 2-fs time step. During these simulations, the pressure and temperature were maintained by using the Langevin algorithm with a coupling constant of 5 ps. SHAKE constraints were applied to all bonds that involved hydrogen atoms (Ryckaert et al, 1977). Random snapshots were extracted along the MD and used to compute the QM/MM emission. These snapshots correspond to low-energy points of the classical MD.

The folding of the C-terminal domain was performed by using umbrella sampling MDs (Kästner, 2011). In detail, the umbrella sampling was realized between the two $\alpha$-carbons of residues 311 and 524. The distance is $21 \AA$ at the beginning and ends at $7 \AA$, with a step of $1 \AA$. For each step, an equilibration of 200 ps followed by a 750-ps production was realized. The lowest energy conformation was also collected for each step. This yields a set of structures along the path that decreases the distance between the two $\alpha$-carbons of residues 311 and 524. The bias introduced by the umbrella potential was removed by using the weighted histogram analysis method (Kumar et al, 1995; Roux, 1995), to generate a free-energy profile along the approach path.

The QM/MM calculations were performed by using a QM/MM coupling scheme between Gaussian (Frisch et al, 2016) and Tinker 
(Tinker, 2005) (Gaussian 09d/Tinker). The electrostatic potential fitted method (Ferré \& Ángyán, 2002) was used to compute the interaction between the Mulliken charges of the QM subsystem and the external electrostatic potential of the MM subsystem within $9 \AA$ from the QM part. The microiterations technique (Melaccio et al, 2011) was used to converge the MM subsystem geometry for every QM minimization step. The emitter was selected as the QM subsystem, whereas the rest of the system was assigned to the MM subsystem. The QM/MM optimization of the first singlet excited state $\left(\mathrm{S}_{1}\right)$ was performed first, followed by calculation of the vertical difference of energies between $S_{1}$ and the ground state $\left(S_{0}\right)$, which corresponds to the fluorescence emission. In fireflies, the fluorescence transition (induced by photoexcitation) is the same as the bioluminescence transition (obtained as a result of a bioluminescence reaction) (Navizet et al, 2013); therefore, the calculated emission value can be equated to the experimental emission energy.

The levels of theory (the treatment of the electron correlation and the basis set) used in the QM of the QM/MM calculations were chosen as follows: the time-dependent density functional theory (TD-DFT) calculations were carried out by using the B3LYP functional with the $6-311 \mathrm{G}(2 \mathrm{~d}, \mathrm{p})$ basis set. The selected basis set was 6$311 \mathrm{G}(2 \mathrm{~d}, \mathrm{p})$; we did not use basis set with diffuse functions because they can interact with the MM system with electrostatic embedding (electrostatic potential fitted method). As detailed in reference (Berraud-Pache \& Navizet, 2016), these conditions are optimal for this type of calculation.

\section{Supplementary Information}

Supplementary Information is available at https://doi.org/10.26508/lsa. 201800072.

\section{Acknowledgements}

This work was supported by New York University (NYU) Abu Dhabi, the Research Enhancement Fund from NYU Abu Dhabi, and Abu Dhabi Education Council. This work was also financially supported by the Human Frontier Science Program (project RGY0081/2011, "Excited-State Structure of the Emitter and Color-Tuning Mechanism of the Firefly Bioluminescence"). The research was partially carried out using the Core Technology Platform resources at NYU Abu Dhabi. We thank the Swiss Light Source (beamline X06SA) and the Australian Synchrotron (beamline MX2) for providing the beamtime for X-ray data collection. I Navizet and R Berraud-Pache acknowledge support from the Agence Nationale de la Recherche (ANR) Biolum project (ANR-16-CE29-0013)

\section{Author Contributions}

C Carrasco-López: data curation, formal analysis, investigation, methodology, project administration, writing-original draft, review, and editing.

JC Ferreira: data curation, formal analysis, and methodology. NM Lui: data curation, formal analysis, and investigation. S Schramm: data curation and formal analysis. $\mathrm{R}$ Berraud-Pache: software and formal analysis.
I Navizet: software, formal analysis, writing-original draft, review, and editing.

$S$ Panjikar: data curation and formal analysis.

P Naumov: conceptualization, funding acquisition, and writingreview and editing.

W Rabeh: supervision, funding acquisition, investigation, methodology, project administration, writing-original draft, review, and editing.

\section{Conflict of Interest Statement}

The authors declare that they have no conflict of interest.

\section{References}

Afonine PV, Grosse-Kunstleve RW, Echols N, Headd JJ, Moriarty NW, Mustyakimov M, Terwilliger TC, Urzhumtsev A, Zwart PH, Adams PD (2012) Towards automated crystallographic structure refinement with phenix.refine. Acta Crystallogr D68: 352-367. doi:10.1107/ s0907444912001308

Alipour BS, Hosseinkhani S, Ardestani SK, Moradi A (2009) The effective role of positive charge saturation in bioluminescence color and thermostability of firefly luciferase. Photochem Photobiol Sci 8: 847-855. doi:10.1039/b901938c

Anandakrishnan R, Aguilar B, Onufriev AV (2012) H++ 3.0: Automating pK prediction and the preparation of biomolecular structures for atomistic molecular modeling and simulations. Nucleic Acids Res 40: W537-W541. doi:10.1093/nar/gks375

Auld DS, Lovell S, Thorne N, Lea WA, Maloney DJ, Shen M, Rai G, Battaile KP, Thomas CJ, Simeonov A, et al (2010) Molecular basis for the highaffinity binding and stabilization of firefly luciferase by PTC124. Proc Natl Acad Sci USA 107: 4878-4883. doi:10.1073/pnas.0909141107

Battye TG, Kontogiannis L, Johnson O, Powell HR, Leslie AG (2011) iMOSFLM: A new graphical interface for diffraction-image processing with MOSFLM. Acta Crystallogr D67: 271-281. doi:10.1107/s0907444910048675

Berraud-Pache R, Navizet I (2016) QM/MM calculations on a newly synthesised oxyluciferin substrate: New insights into the conformational effect. PhysChemChemPhys 18: 27460-27467. doi:10.1039/c6cp02585d

Branchini BR, Murtiashaw MH, Magyar RA, Anderson SM (2000) The role of lysine 529 , a conserved residue of the acyl-adenylate-forming enzyme superfamily, in firefly luciferase. Biochemistry 39: 5433-5440. doi:10.1021/bi9928804

Branchini BR, Southworth TL, Murtiashaw MH, Magyar RA, Gonzalez SA, Ruggiero MC, Stroh JG (2004) An alternative mechanism of bioluminescence color determination in firefly luciferase. Biochemistry 43: 7255-7262. doi:10.1021/bi036175d

Branchini BR, Southworth TL, Murtiashaw MH, Wilkinson SR, Khattak NF, Rosenberg JC, Zimmer M (2005) Mutagenesis evidence that the partial reactions of firefly bioluminescence are catalyzed by different conformations of the luciferase C-terminal domain. Biochemistry 44: 1385-1393. doi:10.1021/bi047903f

Branchini BR, Southworth TL, Fontaine DM, Murtiashaw MH, McGurk A, Talukder MH, Qureshi R, Yetil D, Sundlov JA, Gulick AM (2017) Cloning of the orange light-producing luciferase from Photinus scintillans: A new proposal on how bioluminescence color is determined. Photochem Photobiol 93: 479-485. doi:10.1111/php.12671

Case DA, Cerutti DS, Cheatham TE, Darden TA, Duke RE, Giese TJ, Gohlke H, Goetz AW, Greene D, Homeyer N, et al (2017) AMBER 2017. San Francisco, CA: University of California, San Francisco. 
Chen SF, Liu YJ, Navizet I, Ferré N, Fang WH, Lindh R (2011) Systematic theoretical investigation on the light emitter of firefly. I Chem Theor Comput 7: 798-803. doi:10.1021/ct200045q

Conti E, Franks NP, Brick P (1996) Crystal structure of firefly luciferase throws light on a superfamily of adenylate-forming enzymes. Structure 4: 287-298. doi:10.1016/s0969-2126(96)00033-0

Cruz PG, Auld DS, Schultz PJ, Lovell S, Battaile KP, MacArthur R, Shen M, Tamayo-Castillo G, Inglese J, Sherman DH (2011) Titration-based screening for evaluation of natural product extracts: Identification of an aspulvinone family of luciferase inhibitors. Chem Biol 18: 1442-1452. doi:10.1016/j.chembiol.2011.08.011

DeLano WL (2002) The PyMOL Molecular Graphics System. San Carlos, CA: DeLano Scientific LLC.

DeLuca M (1976) Firefly luciferase. Adv Enzymol Relat Areas Mol Biol 44: 37-68.

Edelheit O, Hanukoglu A, Hanukoglu I (2009) Simple and efficient sitedirected mutagenesis using two single-primer reactions in parallel to generate mutants for protein structure-function studies. BMC Biotechnol 9: 61. doi:10.1186/1472-6750-9-61

Emsley P, Lohkamp B, Scott WG, Cowtan K (2010) Features and development of Coot. Acta Crystallogr D66: 486-501. doi:10.1107/s0907444910007493

Evans P (2006) Scaling and assessment of data quality. Acta Crystallogr D62: 72-82. doi:10.1107/s0907444905036693

Ferré N, Ángyán JG (2002) Approximate electrostatic interaction operator for QM/MM calculations. Chem Phys Lett 356: 331-339. doi:10.1016/s00092614(02)00343-3

Franks NP, Jenkins A, Conti E, Lieb WR, Brick P (1998) Structural basis for the inhibition of firefly luciferase by a general anesthetic. Biophys J 75: 2205-2211. doi:10.1016/s0006-3495(98)77664-7

Frisch MJ, Trucks GW, Schlegel HB, Scuseria GE, Robb MA, Cheeseman JR, Scalmani G, Barone V, Mennucci B, Petersson GA, et al (2016) Gaussian 09, Revision A.02. Wallingford, CT: Gaussian Inc.

Hosseinkhani S (2011) Molecular enigma of multicolor bioluminescence of firefly luciferase. Cell Mol Life Sci 68: 1167-1182. doi:10.1007/s00018010-0607-0

Johnson FH, Shimomura O (1972) Enzymatic and nonenzymatic bioluminescence. Photophysiology 7: 275-334.

Kabsch W (2010) Integration, scaling, space-group assignment and post-refinement. Acta Crystallogr D66: 133-144. doi:10.1107/ s0907444909047374

Kästner J (2011) Umbrella sampling. Wires Comput Mol Sci 1: 932-942. doi:10.1002/wcms.66

Kheirabadi M, Sharafian Z, Naderi-Manesh H, Heineman U, Gohlke U, Hosseinkhani S (2013) Crystal structure of native and a mutant of Lampyris turkestanicus luciferase implicate in bioluminescence color shift. Biochim Biophys Acta 1834: 2729-2735. doi:10.1016/j.bbapap. 2013.09.022

Kumar S, Rosenberg JM, Bouzida D, Swendsen RH, Kollman PA (1995) Multidimensional free-energy calculations using the weighted histogram analysis method. J Comput Chem 16: 1339-1350. doi:10.1002/ jcc.540161104

Melaccio F, Olivucci M, Lindh R, Ferré N (2011) Unique QM/MM potential energy surface exploration using microiterations. Int I Quan Chem 111: 3339-3346. doi:10.1002/qua.23067

Modestova Y, Ugarova NN (2016) Color-shifting mutations in the C-domain of L. mingrelica firefly luciferase provide new information about the domain alternation mechanism. Biochim Biophys Acta 12: 1818-1826. doi:10.1016/j.bbapap.2016.09.007

Nakatsu T, Ichiyama S, Hiratake J, Saldanha A, Kobashi N, Sakata K, Kato H (2006) Structural basis for the spectral difference in luciferase bioluminescence. Nature 440: 372-376. doi:10.1038/nature04542
Navizet I, Liu YJ, Ferré N, Xiao HY, Fang WH, Lindh R (2010) Color-tuning mechanism of firefly investigated by multi-configurational perturbation method. J Am Chem Soc 132: 706-712. doi:10.1021/ ja908051h

Navizet I, Liu YJ, Ferre N, Roca-Sanjuan D, Lindh R (2011) The chemistry of bioluminescence: An analysis of chemical functionalities. ChemPhysChem 12: 3064-3076. doi:10.1002/cphc.201100504

Navizet I, Roca-Sanjuán D, Yue L, Liu YJ, Ferré N, Lindh R (2013) Are the bioand chemiluminescence states of the firefly oxyluciferin the same as the fluorescence state? Photochem Photobiol 89: 319-325. doi:10.1111/ php.12007

Panjikar S, Parthasarathy V, Lamzin VS, Weiss MS, Tucker PA (2005) Auto-rickshaw: An automated crystal structure determination platform as an efficient tool for the validation of an X-ray diffraction experiment. Acta Crystallogr D61: 449-457. doi:10.1107/ s0907444905001307

Panjikar S, Parthasarathy V, Lamzin V, Weiss MS, Tucker PA (2009) On the combination of molecular replacement and single anomalous diffraction phasing for automated structure determination Acta Crystallogr D65: 1089-1097. doi:10.1107/s0907444909029643

Roux B (1995) The calculation of the potential of mean force using computer simulations. Comput Phys Commun 91: 275-282. doi:10.1016/0010-4655 (95)00053-i

Ryckaert JP, Ciccotti G, Berendsen HJC (1977) Numerical integration of the Cartesian equations of motion of a system with constraints: Molecular dynamics of n-alkanes. J Comput Phys 23: 321-341. doi:10.1016/00219991(77)90098-5

Sundlov JA, Fontaine DM, Southworth TL, Branchini BR, Gulick AM (2012) Crystal structure of firefly luciferase in a second catalytic conformation supports a domain alternation mechanism. Biochemistry 51: 6493-6495. doi:10.1021/bi300934s

Tafreshi N, Hosseinkhani S, Sadeghizadeh M, Sadeghi M, Ranjbar B, Naderi-Manesh $\mathrm{H}$ (2007) The influence of insertion of a critical residue (Arg356) in structure and bioluminescence spectra of firefly luciferase. J Biol Chem 282: 8641-8647. doi:10.1074/jbc. m609271200

Thorne N, Shen M, Lea WA, Simeonov A, Lovell S, Auld DS, Inglese J (2012) Firefly luciferase in chemical biology: A compendium of inhibitors, mechanistic evaluation of chemotypes, and suggested use as a reporter. Chem Biol 19: 1060-1072. doi:10.1016/j. chembiol.2012.07.015

Tinker (2005) Software Tools for Molecular Design. Version 5.1. St. Louis, MO: Department of Biochemistry and Molecular Biophysics, Washington University School of Medicine.

Ugarova NN, Brovko LY (2002) Protein structure and bioluminescent spectra for firefly bioluminescence. Luminescence 17: 321-330. doi:10.1002/ bio.688

Vagin A, Teplyakov A (1997) MOLREP: An automated program for molecular replacement. J Appl Crystallog 30: 1022-1025. doi:10.1107/ s0021889897006766

Viviani VR, Bechara EJH, Ohmiya Y (1999) Cloning, sequence analysis, and expression of active Phrixothrix railroad-worms luciferases: Relationship between bioluminescence spectra and primary structures. Biochemistry 38: 8271-8279. doi:10.1021/bi9900830

Viviani VR, Arnoldi FG, Ogawa FT, Brochetto-Braga M (2007) Few substitutions affect the bioluminescence spectra of Phrixotrix (Coleoptera: Phengodidae) luciferases: A site-directed mutagenesis survey. Luminescence 22: 362-369. doi:10.1002/bio.972

Viviani VR, Silva AJ, Arnoldi FGC, Barbosa JARG, Ohmiya Y (2008) The influence of the loop between residues 223-235 in beetle luciferase bioluminescence spectra: A solvent gate for the active site of $\mathrm{pH}$ sensitive luciferases. Photochem Photobiol 84: 138-144. doi:10.1111/ j.1751-1097.2007.00209.x. 


\section{Life Science Alliance}

Viviani VR, Amaral D, Prado R, Arnoldi FG (2011) A new blue-shifted luciferase from the Brazilian Amydetes fanestratus (Coleoptera: Lampyridae) firefly: Molecular evolution and structural/functional properties. Photochem Photobiol Sci 10: 1879-1886. doi:10.1039/ c1pp05210a

Viviani VR, Simões A, Bevilaqua VR, Gabriel GV, Arnoldi FG, Hirano T (2016) Glu311 and Arg337 stabilize a closed active-site conformation and provide a critical catalytic base and countercation for green bioluminescence in beetle luciferases. Biochemistry 55: 4764-4776. doi:10.1021/acs. biochem.6b00260

Winn MD, Ballard CC, Cowtan KD, Dodson EJ, Emsley P, Evans PR, Keegan RM, Krissinel EB, Leslie AG, McCoy A, et al (2011) Overview of the CCP4 suite and current developments. Acta Crystallogr D67: 235-242. doi:10.1107/ s0907444910045749

Wu W, Su J, Tang C, Bai H, Ma Z, Zhang T, Yuan Z, Li Z, Zhou W, Zhang H, et al (2017) cybLuc: An effective aminoluciferin derivative for deep bioluminescence imaging. Anal Chem 89: 4808-4816. doi:10.1021/acs. analchem.6b03510

(c)

License: This article is available under a Creative Commons License (Attribution 4.0 International, as described at https://creativecommons.org/ licenses/by/4.0/). 